\title{
Chemical Imaging Microscopy for Optical Biothreat Detection
}

\author{
P.J. Treado, G.S. Vanni, M.P. Nelson, C. Gardner, J. Wolf, R.S. Schweitzer, J. H. Neiss \\ ChemImage Corporation, 7301 Penn Ave, Pittsburgh, PA 15208
}

Chemical imaging technology combines molecular spectroscopy and digital imaging for rapid, non-invasive, non-contact and reagentless detection of biological warfare agents (BWA). Chemical imaging forms the basis for a new class of optical BWA detection technology, which is made inherently orthogonal by integrating multiple detection strategies into the same system. Modes of specimen interrogation include laser excited Raman scattering and fluorescence emission combined with digital imaging [1]. Each of these detection strategies operating independently provides a limited degree of sensitivity and specificity. By combining each of these detection modalities it is being shown that sensitivity and specificity increase.

Chemical imaging technology resolves objects less than a micron in size and can do so in the presence of interfering materials such as encapsulating glass or plastic, food, water or other fluids, including bodily fluids, and environmental contaminants. Species classification and identification is performed by electronically querying a database of spatial and spectral signatures that describe the unique morphologies and molecular properties of threat species. Chemical imaging technology can simultaneously test for the presence of numerous threat agents, and adding database entries to detect new threat agents is a straightforward process that does not require significant sample preparation or handling.

Chemical imaging enables spatial-specific spectra to be collected that are then used to unravel composite chemical signatures present in complex mixtures. This technique employs the use of multivariate statistical analysis routines, generally known as chemometrics, to further aid in the interpretation of complex mixtures even when multiple components are co-localized. Such tools can greatly reduce the incidence of false positives and false negatives and can provide a method to determine within seconds to minutes if a biothreat is present.

For BWA detection, fluorescence spectroscopy is particularly useful for rapid screening of suspect biotic material. Raman spectroscopy is useful because each specimen exhibits a characteristic 'fingerprint' spectrum, resulting from various selection rules. Peak intensity, shape and position are used to determine molecular composition, conformation (crystalline phase, degree of order, strain, grain size, etc.) and concentration. The high degree of specificity of Raman spectroscopy is well known. Whereas traditional detection methods are not generally capable of detecting and identifying bacterial species at the single spore level, chemical imaging can be used to "fingerprint" bacterial spore types down to the single-spore level.

Figure 1 shows fluorescence chemical imaging results for a mixture of bacterial endospores, namely Bacillus globigi (BG) and Bacillus stearothremophilus (BS). Fluorescence spectra (Fig. 1A), optical images (Fig. 1B), and fluorescence chemical images (Fig. 1C) are collected on a chemical imaging microscope (FALCON, ChemImage Corporation) having fluorescence and Raman detection capability [2]. The broad fluorescence emission spectra exhibited by the endospores are efficiently resolved within seconds on a pixel by pixel basis using the chemical 
A

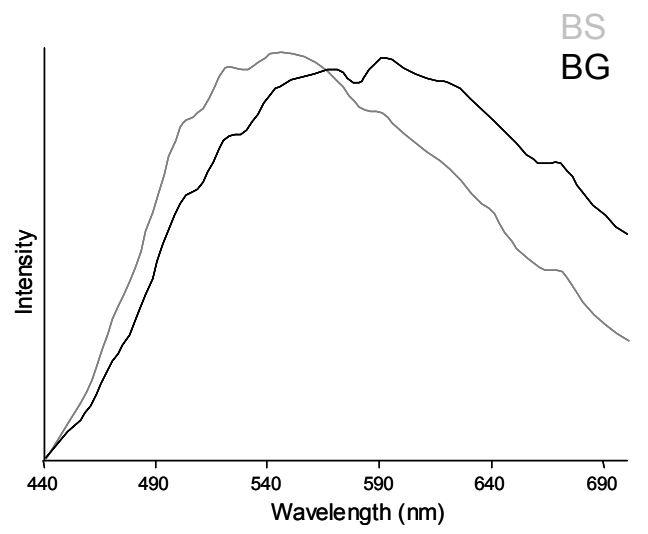

B

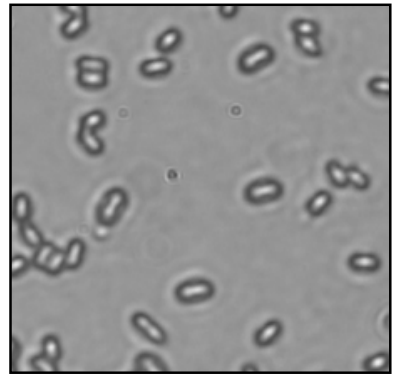

C

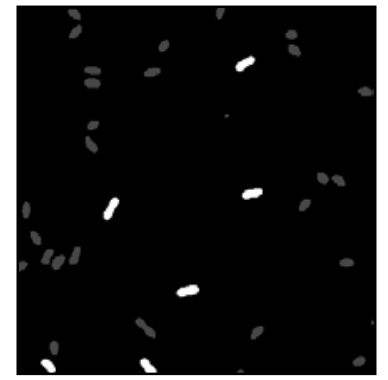

Figure 1 Fluorescence chemical imaging of a mixture of bacterial endospores, Bacillus globigi (BG) and Bacillus stearothremophilus (BS). (A) Visible fluorescence emission spectra of BG and BS under uv (360-380 nm) excitation. (B) Optical micrograph (100X dry objective magnification, $0.95 \mathrm{NA}$ ) of mixture. (C) Chemical image discrimination of BG (white) vs BS (grey).

imaging microscope system. The resulting spectra and images associated with each endospore object are analyzed on the basis of the morphology (size and shape distribution) and spectral patterns they exhibit. As a result, the biological agents are discriminated with a higher degree of sensitivity and specificity than if morphology or spectral patterns were used independently.

\section{References}

1. Treado, P. J. and Nelson, M. P. "Raman Imaging” in Handbook of Vibrational Spectroscopy, Vol. 2: Eds. Chalmers, J. M. and Griffiths, P. R., pp 1429-1459 (John Wiley \& Sons, LTD) 2002.

2. Morris, H. R., Turner, J. F., Munroe, B., Ryntz, R. A. and Treado, P. J. "Chemical Imaging of Thermoplastic Olefin (TPO) Surface Architecture," Langmuir 15, 2961 (1999). 